\title{
Reglamento de publicación
}

La Revista de la Asociación Argentina de Ortopedia y Traumatología (AAOT) publica en forma exclusiva las comunicaciones y relatos (ambos inéditos) leídos en las sesiones científicas de la AAOT, los trabajos también inéditos aportados por las sociedades y capítulos integrantes de Asociación, los trabajos y comunicaciones de los congresos de la AAOT y los trabajos enviados para su eventual publicación.

El Consejo de Redacción selecciona los trabajos y los envía en forma anónima a la Revisión por los Pares (referato), quienes los aceptan con modificaciones o no, o los rechazan. El Consejo de Redacción remite a los autores los trabajos ya aceptados que necesitan correcciones y se reserva el derecho de publicarlos en forma completa o resumida.

Los trabajos presentados en las sesiones científicas de la AAOT no seleccionados para su publicación completa en la Revista serán publicados a modo de resúmenes.

La AAOT no se hace responsable de los conceptos vertidos por los autores o anunciantes en la Revista.

Todos los autores de los artículos presentados para la publicación en la Revista deben firmar la carta referida a la cesión de los derechos de autor.

\section{Instrucciones para los autores}

\section{Información general}

La Revista de la Asociación Argentina de Ortopedia y Traumatología publica artículos originales, revisados por pares, relacionados con el diagnóstico, tratamiento y fisiopatología de las enfermedades y las condiciones del aparato locomotor, las cuales incluyen tanto los estudios de ciencia básica y clínica, junto con los reportes de casos.

Los artículos aceptados serán publicados en la revista impresa, así como en la versión en línea. Los materiales suplementarios aceptados con el manuscrito que no puede ser publicado en la versión impresa, debido a las limitaciones de espacio o medio puede aparecer en la versión en línea, este material se citará en el texto del artículo y el lector se dirigirá a la revista en línea.

El material que requiera un trabajo editorial importante se devolverá sin revisión para su corrección previa a la revisión.

La Revista utiliza una revisión por pares anónima en la evaluación de manuscritos para su publicación.

Los autores deben enviar los manuscritos electrónicamente a través del nuevo sistema editorial: http://ojs.aaot.org.ar

\section{Política editorial}

Las afirmaciones y opiniones expresadas en los artículos y las comunicaciones son las del autor(es) y no necesariamente las del editor o co-editor, o la Asociación Argentina de Ortopedia y Traumatología (AAOT). Declinamos toda responsabilidad por dicho material, ni respaldamos ningún producto o servicio anunciado en esta publicación. Tampoco responden por cualquier demanda o reivindicación efectuada por el fabricante de dicho producto o servicio.

\section{Derecho de autor (copyright)}

Todo el material publicado en el Revista de la Asociación Argentina de Ortopedia y Traumatología está cedido a la Asociación Argentina de Ortopedia y Traumatología (AAOT). De conformidad con la Ley de Derecho de Autor (Ley 11.723), al autor correspondiente de cada manuscrito se le pedirá que complete un formulario de cesión de derechos de autor sobre la aceptación del manuscrito. Al enviar un artículo el autor(es) debe hacer una declaración completa al editor sobre todas las presentaciones e informes previos que pudieran considerarse como publicación previa o duplicada del mismo trabajo o muy similares. Copias de dicho material debe ser incluido en el documento presentado para ayudar al editor a decidir cómo tratar el asunto.

\section{Investigación en seres humanos}

Los artículos que implican la investigación realizada en seres humanos deben incluir una declaración en la sección de Materiales y Métodos que indica la aprobación del Comité de Ética Institucional o autoridad competente y tomando nota de que el consentimiento informado, así como cualquier consentimiento necesario, se obtuvo de 
cada paciente. Para los informes de la investigación con sujetos humanos, dar garantías de que: (a) el consentimiento necesario y apropiado se ha obtenido de cada paciente y (b) el protocolo de estudio se realizó conforme a las normas éticas de la Declaración de Helsinki de 1975 como se refleja en la aprobación previa del Comité de Ética Institucional. Pacientes individuales deben ser referidos por número, no por las iniciales.

\section{Experimentación con animales}

Los manuscritos que reporten experimentos con animales deben incluir una declaración en la sección de Materiales y Métodos que el cuidado de los animales cumplen con las directrices de la institución de los autores y los Institutos Nacionales de Salud y cualquier ley nacional sobre el cuidado y uso de animales de laboratorio.

\section{Permisos}

Citas directas, tablas o ilustraciones tomadas de material con derechos de autor deben ser acompañados de un permiso para su uso, del propietario del copyright original. El permiso se presenta como una nota al pie de texto o una tabla o como una adición a la leyenda de la figura y debe proporcionar información completa de la fuente. Las fotografías de personas identificables deben ir acompañada de un comunicado firmado que indica el consentimiento informado.

\section{Unidades de medida}

Utilice el Sistema Internacional (SI) de Unidades.

\section{Abreviaciones}

Se escribe una vez completa luego entre paréntesis la abreviatura y en adelante se la utiliza en el texto.

\section{Nombres de los medicamentos e implantes}

Utilice los nombres genéricos. El nombre del fabricante se puede mencionar entre paréntesis, junto con la ciudad y país.

\section{Los manuscritos revisados}

Cada manuscrito revisado debe ir acompañado de una carta de presentación que se ocupa de cada uno de los comentarios de los revisores. Esta carta debe detallar punto por punto que se han producido cambios y, las razones para los cambios recomendados que no se han hecho. Cada cambio debe ir acompañado del número de línea, párrafo y la página donde fue realizado en el artículo revisado. El número del manuscrito debe aparecer en la carta de presentación.

Los Autores que deseen publicar en la revista deberán seguir las siguientes instrucciones. Todos los manuscritos serán presentados electrónicamente en el sitio ONLINE de la revista http://ojs.aaot.org.ar; donde se deberá registrar como autor y será guiado paso a paso para cargar su manuscrito. En el sitio web podrá seguir el progreso del manuscrito. Comunicaciones acerca del manuscrito serán enviadas por e-mail.

\section{Número de autores}

El orden de los autores sólo refleja la preferencia de los autores. Cada autor debe haber contribuido de manera significativa, y estar dispuestos a tomar responsabilidad pública por, uno o más aspectos del estudio: su diseño, adquisición de datos y el análisis e interpretación de datos. Todos los autores deben haber participado activamente en la redacción y revisión crítica del manuscrito, y cada uno debe dar la aprobación final de la versión para ser publicada. Las personas que han contribuido a una sola sección del manuscrito o han contribuido solo al aporte de casos deben ser mencionados en una nota de agradecimiento.

Si un grupo de investigación ha sido designado como el autor de un artículo, uno o más miembros del grupo que responden plenamente a los criterios anteriores de la autoría deben ser listados como autores del artículo, seguido de "en nombre de [nombre del grupo]." Los otros miembros del grupo deben figurar en una sección de agradecimientos al final del artículo. Por otra parte, en la lista de autores también se puede incluir sólo el nombre del grupo, seguido de un asterisco que corresponde a una lista que especifica los autores que responden plenamente a los criterios anteriores para la autoría y que también menciona a otros miembros del grupo.

El manuscrito original debe ser escrito en computadora en formato Word a doble espacio con letras Times New Roman tamaño número 12 con márgenes amplios de $3 \mathrm{~cm}$. Las páginas deben ser numeradas y las tablas y leyendas de figuras deben ir separadas en páginas separadas del texto principal. 
Las imágenes deben enviarse como archivo adjunto en JPG y con la referencia en un formato Word también como archivo adjunto independiente, por ejemplo al mandar una imagen se debe enviar el archivo en formato JPG con nombre de archivo figura 1 y se manda un archivo adjunto en formato Word con nombre de referencia de Figura 1 donde se explica o detalla la figura 1.

\section{Preparando el manuscrito}

El nombre de los autores completos con su grado (ejemplo: Dr. o Licenciado en rehabilitación, etc), con sus referencias institucionales (lugar de trabajo, dirección y localización con estado y país) y los datos de correspondencia (nombre, dirección, número de teléfono y/o fax, y dirección de e-mail) serán cargados duran te el proceso de envío del manuscrito en el OJS. Si lo tuviere, es imprescindible incluir en la carga del OJS información del soporte financiero del trabajo a publicar. En caso negativo, también debe figurar. Ud. Puede solicitar un "Tutorial" para la carga de su artículo a publicaciones@aaot.org.ar.

El título debe ser escogido con sumo cuidado: si es corto tiene mayor impacto. Las palabras utilizadas en el mismo deben facilitar la búsqueda del tema tratado en un índice bibliográfico siendo lo mas adecuado el uso de palabras clave, para su ubicación mediante buscadores.

La primer página del artículo debe incluir: el título y el resumen en español y en ingles.

El resumen debe tener como máximo 250 palabras y deberá ser enviado en castellano y en inglés, precederá al artículo; debiendo resumir y resaltar los puntos más importantes del mismo. Estructuralmente deberá incluir objetivo, material y métodos, resultados, conclusiones, nivel de evidencia.

Las palabras claves que no deberán ser más de 6, deberán incluirse al final del resumen en español y en inglés.

En la página siguiente página comienzo el texto propiamente dicho. Por favor en el texto no incluir ninguna identificación (ejemplo: nombres de autores o nombre de institución donde se realizó el estudio, dirigirse en el texto por ejemplo como el cirujano autor o como nuestra institución, etc).

\section{El texto}

Será dividido en cuatro secciones: Introducción, Material y métodos, Resultados y Discusión. Con el fin de mantener la coherencia del mensaje, se debe pensar cada sección en relación con la pregunta o hipótesis del trabajo. De esta manera, la Introducción formula la pregunta, en Material y métodos se describen las actividades realizadas para responder dicha pregunta, en Resultados se reportan los resultados obtenidos, y en Discusión se plantea las diferencias y/o similitudes con otros autores, las consideraciones del autor y, finalmente, se responde la pregunta formulada.

Introducción: Se plantea la justificación del tema que lleva a formular el objetivo. Se debe mencionar solo las citas bibliográficas indispensables. Y concluir la introducción postulando el objetivo y/o la hipótesis del estudio.

Material y métodos: Su función es describir las actividades realizadas para responder la pregunta planteada en la introducción. Debe proveer suficiente información para permitir a otro científico evaluar la credibilidad del trabajo y repetir el experimento tal cual fue realizado. Debe describir claramente la población de estudio, los métodos para su evaluación así como aparatos y procedimientos utilizados para permitir al lector reproducir los mismos resultados. Esta sección no debe incluir resultados. Los datos pueden ser presentados en tablas. Definir el tipo de diseño del estudio y describir el método estadístico utilizado. En esta sesión debe constar que el estudio fue aprobado por un comité evaluador o de ética y de que institución.

Resultados: Se presentan los resultados obtenidos según se describió en la sección material y métodos. Si los resultados se presentan en tablas deben resumir y enfatizar los resultados más importantes, no repetir los datos del texto.

Discusión: Tiene como objeto describir los resultados obtenidos respondiendo al objetivo. Aclarar las limitaciones del estudio, como las coincidencias o disidencias halladas en la literatura. Definir la repercusión que los resultados pueden tener no solo según la hipótesis sino también a otro nivel de conocimiento, para dar iniciativa a otros trabajos.

Las presentaciones de casos y los trabajos para las secciones de Actualización e Instrucción ortopédica de posgrado no llevan resúmenes.

Para los estudios clínicos científicos, incluya el tipo de estudio y el nivel de evidencia (véase el cuadro) al final del resumen.

Referencias: Los autores son responsables de verificar la exactitud e integridad de las referencias. Las referencias no deben ser simplemente una lista de los resultados de una búsqueda bibliográfica informatizada, sino que deben haber sido leídos por el autor(es) y se consideran pertinentes para el manuscrito. Escriba las referencias 
con doble espacio en hoja separada del texto y enumérelas consecutivamente por el orden en que fueron citadas en el texto. Cite las referencias en el texto mediante números arábigos entre paréntesis. Incluya todos los autores cuando sean 6 o menos, cuando sean 7 o más, mencione los 6 primeros y agregar et al. Utilice las abreviaturas de títulos de revistas según el Index Medicus. Incluya los números de página de inicio y final de cada cita. Incluya el DOI, si corresponde. Si una fuente de referencia no se ha publicado todavía, pero ha sido aceptada para su publicación, incluya la fuente en la lista de referencia y presentar la carta de aceptación junto con el manuscrito.

Se insta a los autores a citar todos los trabajos publicados previamente en la Revista de la AAOT, que hagan referencia a la investigación. Para poder contar con estos artículos, se sugiere realizar una búsqueda bibliográfica en: http://lilacs.bvsalud.org/es/ o solicitar tutorial para la búsqueda a través de la biblioteca de la AAOT: biblioteca@ aaot.org.ar

No citar resúmenes de congresos, comunicaciones personales o material no publicado (incluyendo presentaciones orales, y manuscritos aún no aceptados para su publicación) en la lista de referencias. Si la información es fundamental para el mensaje del manuscrito, este material puede ser identificado en el texto entre paréntesis.

Por favor, tenga en cuenta los siguientes ejemplos de estilo de referencia.

Artículo de revista: Saunders RA, Frederick HA, Hontas RB. The Sauve'- Kapandji procedure: a salvage operation for the distal radioulnar joint. J Hand Surg 1991;16A:1125-29.

Libro: Taleisnik J. The wrist. New York: Churchill Livingstone;1985. p. 25-32.

Capítulo de libro: Bowers WH. The distal radioulnar joint. In: Green DP, ed. Operative hand surgery. $3^{\text {rd }}$ ed. New York: Churchill Livingstone; 1993. p. 973-1020.

\section{Tablas}

Las tablas se deberán presentar en formato Word (no se aceptarán otros formatos), a doble espacio, con número y título. Las notas explicativas se deben escribir a continuación del margen inferior de la tabla. Cada tabla debe estar en una página aparte. No se debe duplicar la información de las tablas con el texto o las figuras.

\section{Leyendas de figuras}

Enumere las figuras con números arábigos en el orden mencionado en el texto. Proporcionar una explicación suficiente para que la figura sea comprensible sin tener que referirse al texto. Defina todos los símbolos y todas las abreviaturas que aún no ha escrito en el texto. Para cualquier material con derechos de autor, indicar que ha obtenido el permiso (permisos, ver más arriba). Las leyendas deben ser escritas consecutivamente en una página separada del cuerpo del manuscrito.

\section{Figuras}

Las ilustraciones digitales serán aceptadas con la mayor resolución y nitidez posible en colores y en formato TIFF o JPEG. Deben estar correctamente identificadas y enumeradas (p. ej. Figura 1.jpeg, Figura 2A.jpeg). El tamaño máximo de cada archivo que aceptará el sistema es de 4MB (megabytes). Sin embargo para una velocidad mayor de navegación y cargado de los archivos, se sugiere un tamaño de $2 \mathrm{MB}$ por figura.

Utilice las flechas de producción profesional u otros marcadores colocados directamente sobre la figura para identificar las características importantes. Recortar figuras como sea necesario sin que pierda el significado o la información a mostrar. Indique la parte superior de la fotografía en caso de que la orientación no sea evidente. Los nombres de los autores o los nombres de las instituciones no deben aparecer en ninguna parte de las figuras. El título de la figura y el material de leyenda aparecen en la leyenda, no en la figura. Los números de la figura debe corresponder con el orden en que las presentan en el texto. Si se usan fotografías de personas, los sujetos no deben ser identificables o se debe presentar una autorización por escrito de la persona para utilizar la fotografía. Si una figura ya fue publicada, se debe reconocer la fuente original y presentar la autorización escrita del titular del copyright para reproducir el material.

\section{Videos}

La incorporación de videos a los artículos será opcional. Se aceptará sólo uno por artículo. No podrá pesar más de 100 MB y no debe durar más de 4 minutos. Debe contener subtítulos que vayan describiendo la técnica utilizada o puede ser narrado si el autor así lo prefiere (¡los animamos a eso!).

El video debe ser citado en el texto del manuscrito (video). La Revista no se encarga de la edición del video. 


\begin{tabular}{|c|c|c|c|c|}
\hline \multicolumn{5}{|c|}{ TIPO DE ESTUDIOS } \\
\hline $\begin{array}{c}\text { Nivel } \\
\text { de } \\
\text { evidencia }\end{array}$ & $\begin{array}{l}\text { Estudios terapéuticos: } \\
\text { investigan resultados } \\
\text { de tratamientos }\end{array}$ & $\begin{array}{l}\text { Estudios pronóstico: } \\
\text { investigan el efecto } \\
\text { de una característica } \\
\text { del paciente y evalúan } \\
\text { el resultado de la } \\
\text { patología }\end{array}$ & $\begin{array}{l}\text { Estudios diagnósticos: } \\
\text { investigan un método } \\
\text { diagnóstico }\end{array}$ & $\begin{array}{l}\text { Análisis económico: } \\
\text { desarrollo de modelo } \\
\text { económico o de } \\
\text { indicación }\end{array}$ \\
\hline Nivel I & $\begin{array}{l}\text { - Estudio aleatorizado con } \\
\text { grupo de control de alta } \\
\text { calidad, con diferencia } \\
\text { estadísticamente } \\
\text { significativa o no, pero } \\
\text { con mínimo intervalo de } \\
\text { confidencia } \\
\text { - Revisión sistemática } \\
\text { de estudios de nivel I } \\
\text { con grupo de control } \\
\text { aleatorizado }\end{array}$ & $\begin{array}{l}\text { - Estudio prospectivo de } \\
\text { alta calidad (todos los } \\
\text { pacientes se incluyen } \\
\text { en el mismo punto de } \\
\text { la patología y el } 80 \% \\
\text { de los pacientes deben } \\
\text { estar incluidos en el } \\
\text { seguimiento) } \\
\text { - Revisión sistemática de } \\
\text { estudios de nivel I }\end{array}$ & $\begin{array}{l}\text { - Estudios de criterios } \\
\text { diagnósticos ya } \\
\text { descritos en una } \\
\text { serie consecutiva de } \\
\text { pacientes (con un } \\
\text { universo de referencia } \\
\text { gold standard) } \\
\text { - Revisión sistemática } \\
\text { de estudios de nivel I }\end{array}$ & $\begin{array}{l}\text { - Estudios costo- } \\
\text { sensibles y } \\
\text { alternativas; valores } \\
\text { obtenidos de varios } \\
\text { estudios; múltiples } \\
\text { formas de análisis de } \\
\text { sensibilidad } \\
\text { - Revisión sistemática } \\
\text { de estudios de nivel I }\end{array}$ \\
\hline Nivel II & $\begin{array}{l}\text { - Estudio aleatorizado con } \\
\text { grupo de control } \\
\text { de menor calidad (p. ej., } \\
<80 \% \text { de seguimiento } \\
\text { en los pacientes } \\
\text { incluidos, no ciegos } \\
\text { o una aleatorización } \\
\text { inadecuada) } \\
\text { - Estudio prospectivo } \\
\text { comparativo } \\
\text { - Revisión sistemática de } \\
\text { estudios de nivel II o } \\
\text { estudios de nivel I con } \\
\text { resultados inconsistentes }\end{array}$ & $\begin{array}{l}\text { - Estudios retrospectivos } \\
\text { - Grupo no tratado de } \\
\text { un grupo de control } \\
\text { aleatorizado } \\
\text { - Estudio prospectivo de } \\
\text { menor calidad (p. ej., < } \\
\text { 80\% de seguimiento en } \\
\text { los pacientes incluidos } \\
\text { o pacientes incluidos en } \\
\text { diferentes estadios de la } \\
\text { patología) } \\
\text { - Revisión sistemática de } \\
\text { estudios de nivel II }\end{array}$ & $\begin{array}{l}\text { - Desarrollo de criterio } \\
\text { diagnóstico en una } \\
\text { base consecutiva de } \\
\text { pacientes (con un } \\
\text { universo de referencia } \\
\text { gold standard) } \\
\text { - Revisión sistemática } \\
\text { de estudios de nivel II }\end{array}$ & $\begin{array}{l}\text { - Estudios costo- } \\
\text { sensibles y } \\
\text { alternativas; valores } \\
\text { obtenidos de estudios } \\
\text { limitados; múltiples } \\
\text { formas de análisis de } \\
\text { sensibilidad } \\
\text { - Revisión sistemática } \\
\text { de estudios de nivel II }\end{array}$ \\
\hline Nivel III & $\begin{array}{l}\text { - Estudio de casos y } \\
\text { controles } \\
\text { - Estudio retrospectivo } \\
\text { comparativo } \\
\text { - Revisión sistemática de } \\
\text { estudios de nivel III }\end{array}$ & - Estudio de caso control & $\begin{array}{l}\text { - Estudio de pacientes } \\
\text { no consecutivos } \\
\text { (sin un universo } \\
\text { de referencia gold } \\
\text { standard) } \\
\text { - Revisión sistemática } \\
\text { de estudios de nivel } \\
\text { III }\end{array}$ & $\begin{array}{l}\text { - Análisis basados en } \\
\text { costos y alternativas } \\
\text { limitadas, pobre } \\
\text { estimación } \\
\text { - Revisión sistemática } \\
\text { de estudios de nivel } \\
\text { III }\end{array}$ \\
\hline Nivel IV & - Serie de casos & - Serie de casos & $\begin{array}{l}\text { - Estudio de casos y } \\
\text { controles } \\
\text { Pobre referencia } \\
\text { estándar }\end{array}$ & - Análisis no sensitivo \\
\hline Nivel V & Opinión de expertos & Opinión de expertos & Opinión de expertos & Opinión de expertos \\
\hline
\end{tabular}

Traducción del original en inglés. Instructions for Authors. En: J.Bone Joint Surg.Am [en línea] <http://jbjs.org/public/instructionsauthors.aspx> 